\title{
Bio-Relevant and Bioequivalence Studies: An Overview
}

\section{Suryakanta Swain* and Nerella Nagadivya}

Department of Pharmaceutics, Roland Institute of Pharmaceutical Sciences, India

\section{Importance of Open Access and Special Features of OMICS Group}

Open access plays a pivotal role in propagating scientific information globally. It is completely user friendly and is open for all to access easily. Its salient features are, that it covers 50 world's leading languages and have the facility of audio versions too. It invites every individual to share as well as explore scientific knowledge and research in the field of pharmaceutical science. As it is similar to social networking it meets the requirements of young generation and provides an excellent platform for new pharma scholars. Share important information about innovative technologies and other regulatory affairs. It enhances scientific skills of the authors, readers, reviewers, editors and advisors by providing scientific credits. On whole it encourages each and everyone and attracts all towards the majestic world of science.

\section{Structure of the Editorial}

Statistical studies include the study of bio relevant media which are biologically relevant and reflect what's actually inside our bodies. These medias mimic the body fluids such as bile salts, lethicin, bile juice etc which are absent in conventional test media. Most of the drugs are consumed orally so, they must possess excellent dissolution rate and adsorption properties in the human gastro intestinal tract (GIT). But the problem associated with conventional test media is that, they don't mimic the highly variable and dynamic environment of the human GIT. To overcome this above problem bio relevant medias have come into action. Testing the drugs in them can help us to predict how an oral drug is likely to dissolve and release in vivo far more accurately and its performance in patients. There are many benefits through bio relevant studies such as effective formulation development in pharmaceutical industries, to match the in vitro dissolution profile of the test product with the innovator in context with generic industries, only a few experiments would be conducted on animals and better medicines are designed and formulated for patients. For conducting bio relevant studies we need suitable bio relevant media which can be easily prepared in seconds by simply adding the Sif powder with that of the prepared buffer solution. The Sif powder is cost effective as well as time saving and most importantly reproducible one. It originally makes three different kinds of bio relevant medias; fassif, fessif and fassgf. It's a patented complex of bile salts (sodium taurocholate) and lethicin (phospholipids) in 4:1 molar ratio based on Professor Jennifer Dressman's original formulation. Both bioequivalent and bio relevant studies are quite useful for formulation of better drug systems by determining its pharmacological response outside the body which is similar to human viscera. It is ready to lend a hand in the characterization of complex drug substances and products, expanding biopharmaceutics, classification of systemic biowaivers, statistical methods for drug profile comparisons, novel phamacodynamic study designs and food and drug interactions etc. more importantly, it will expand the range of products for which generic versions are available meanwhile maintaining high standards of quality, safety and efficacy.

Bioequivalence means two pharmaceutical products are said to be bioequivalent if their bioavailability are equal, dose is same, route of administration is same, therapeutic effect with respect to both safety and efficacy is equal meeting same comparable standards. According to FAD the definition of bioequivalence is the absence of significant difference in rate and extent to which API or active moiety in pharmaceutical equivalent becomes available at the site of drug action when administered in similar conditions. This is a technique which involves the different pharmacokinetics parameters like $\mathrm{C}_{\max }$, $\mathrm{T}_{\max }, \mathrm{T}_{\log }$ and AUC etc. If pharmacokinetic studies are not feasible then, pharmacodynamic criteria for studying drug comes into picture. Study of bioequivalence is necessary to observe the adsorption of drug into the GIT, which can be achieved by in vitro (dissolution or assay) and in vivo (study of pharmacokinetic properties or assay) methods. It further ensures the switch ability of patient's medication. Apart from these, bioequivalent study deals with the parametric and non parametric statistical techniques to test the drugs. The possible distinction between the branded and generic products is evaluated through bioequivalence approach.

On behalf of the editor and editorial board member for this journal, I would like to thank the OMICS publishing group 5716 Corsa Ave, Suite 110 Westlake, Los Angeles, A 91362-7354, USA for giving me another opportunity for writing an invited editorial article for publishing the upcoming issue with my co-author.
*Corresponding author: Suryakanta Swain, Department of Pharmaceutics, Roland Institute of Pharmaceutical Sciences, Berhampur-760 010, Odisha, India, Tel: +919438038643; Fax: +91-680-2404112; E-mail: swain_suryakant@yahoo.co.in

Received September 02, 2014; Accepted September 03, 2014; Published September 05, 2014

Citation: Swain S, Nagadivya N (2014) Bio-Relevant and Bioequivalence Studies: An Overview. Pharmaceut Reg Affairs 3: e140. doi:10.4172/2167-7689.1000e140

Copyright: ( 2014 Swain S, et al. This is an open-access article distributed under the terms of the Creative Commons Attribution License, which permits unrestricted use, distribution, and reproduction in any medium, provided the original author and source are credited. 